\title{
2:1 Atrioventricular Conduction due to Congenital Long QT Syndrome
}

\author{
Maneesh K. Rai ${ }^{1}$ Soma Sekhar Ghanta ${ }^{2}$ \\ ${ }^{1}$ Department of Cardiology, Kasturba Hospitals, \\ Mangalore, Karnataka, India \\ ${ }^{2}$ Department of Electrophysiology, Aayush Hospitals, \\ Vijayawada, Andhra Pradesh, India \\ Indian J Cardiovasc Dis Women-WINCARS 2017;2:54-55.
}

\begin{abstract}
Address for correspondence Soma Sekhar Ghanta, MD, DM, FEp Department of Electrophysiology, Aayush Hospitals, Vijayawada 520008, Andhra Pradesh, India (e-mail: somasekharghanta1978@gmail.com).
\end{abstract}

\author{
Abstract \\ Long QT syndrome patients can present as second-degree AV block due to prolongation \\ of refractory period. We report a case of pseudo 2:1 AV block due to long QT syndrome \\ Keywords \\ - long QT \\ - second-degree AV \\ block \\ - 2:1 AV conduction
}

\section{Introduction}

Congenital long QT syndrome is a rare disorder of prolongation of ventricular refractory period with prevalence of 1 in 5,000. It is a channelopathy with autosomal dominant and recessive inheritance with potassium, sodium, and calcium channel abnormalities. Patients with this disorder can present with syncope, ventricular tachyarrhythmias, and sudden cardiac arrest.

Electrocardiographic (ECG) manifestations of long QT syndrome are prolonged QT more than 470 milliseconds, T wave alternans, polymorphic ventricular tachycardia (VT), and, rarely, 2:1 atrioventricular (AV) conduction..$^{1-3}$

\section{Case Report}

A 3-month-old child was referred to us with a history of shortness of breath for cardiac evaluation. She was evaluated with ECG revealing prolonged QT with QTc of 440 milliseconds and 2:1 AV conduction( - Fig. 1A). Echocardiogram was normal with no evidence of structural abnormalities. Chest radiograph was also nondistinct. With suspicion of long QT, evaluation of sibling of 1 year 6 months was done, which revealed prolonged QT interval with QTc of 480 milliseconds ( - Fig. 1B), suggesting familial long QT syndrome. There was no family history of sudden cardiac arrest or syncope in siblings. She was started on propranolol for treatment of long QT and followed up.

\section{Discussion}

Long QT syndrome is an inherited disorder of cardiac ion channel associated with prolongation of ventricular refractory period. It has predisposition to life-threatening ventricular arrhythmia causing sudden death. It can present as palpitations, presyncope, and aborted sudden cardiac arrest due to lifethreatening ventricular arrhythmia predominantly polymorphic VT. When ventricular refractory period is extremely prolonged more than sinus rates, it may present as 2:1 AV conduction. When refractory period of ventricular myocardium is prolonged with preserved rate-dependent abbreviation of refractory period of Purkinje's network, sinus tachycardia manifests as 2:1 AV conduction. ${ }^{2}$ Though prolonged QTc is taken as 470 milliseconds as per Schwartz study, there are studies reported with mean QTc less than 440 milliseconds in Indian newborn infants. ${ }^{4}$ In our case, corrected QTc is 440 milliseconds and PP interval is also 440 milliseconds, which is relatively prolonged causing $2: 1 \mathrm{AV}$ conduction. Here $\beta$-blockers are mainstay of therapy in long QT, unlike other causes of 2:1 $\mathrm{AV}$ conduction. Genomic analysis is important in evaluating pediatric patients with suspicion of long QT syndrome for genetic counseling and guiding therapy as there is type-specific intervention, with long QT types 1 and 2 responding better to $\beta$ blockers and type 3 responding better to mexiletine and, rarely, requiring pacing. ${ }^{1}$ 
A
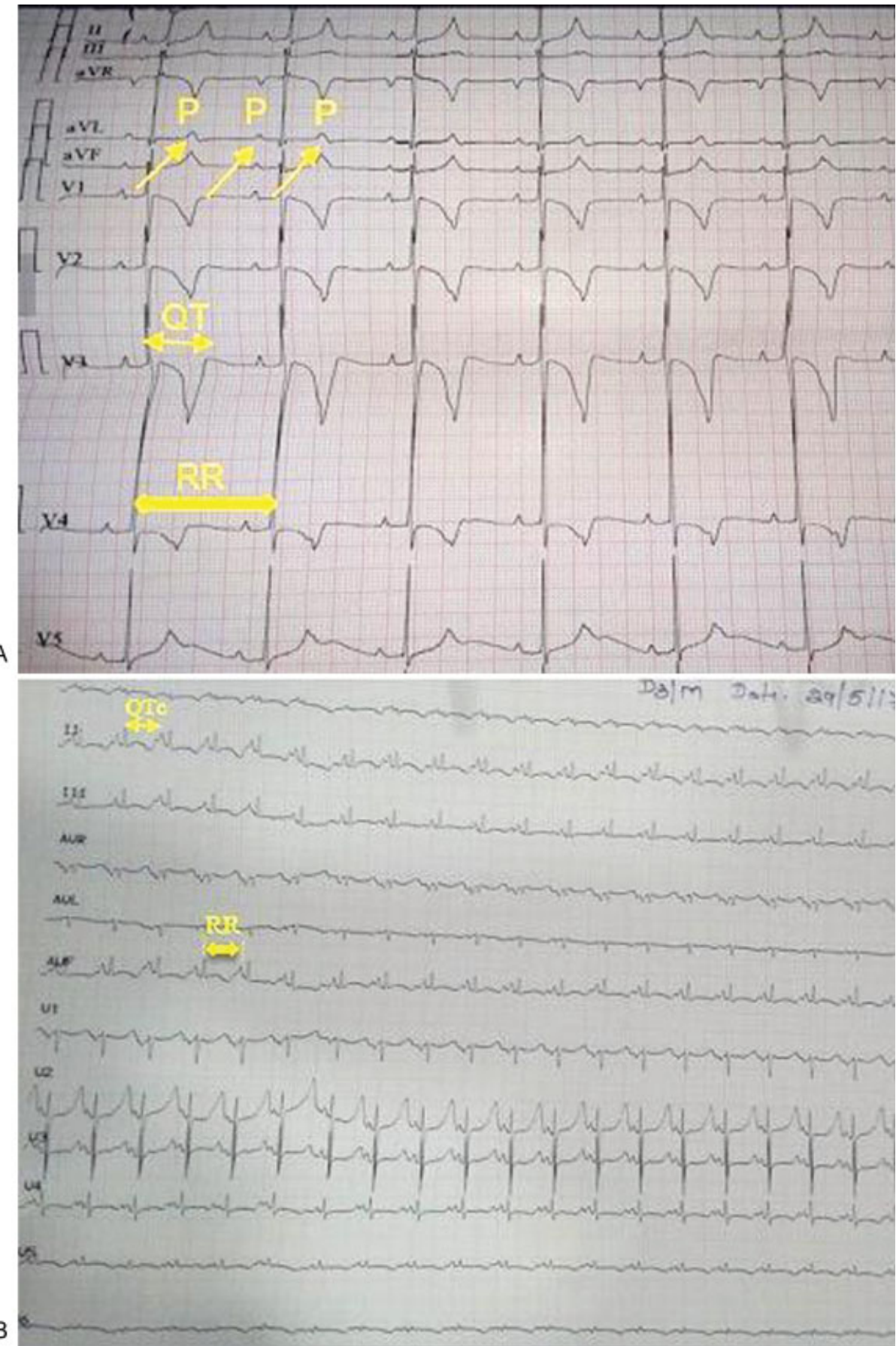

Fig. 1 (A) ECG of index patient showing arrows pointing $P$ waves with alternate $P$ on T waves and QT of 440 milliseconds and corrected QTc of 440 milliseconds. (B) ECG of sibling showing sinus tachycardia with RR interval of 440 milliseconds, QT interval of 320 milliseconds with QTc of 480 milliseconds.

\section{References}

1 Cevik BS, Cevik A, Tavli E. Second degree heart block associated with QT prolongation. Indian Pacing Electrophysiol J 2010;10(02):96-98 2 Rosenbaum MB, Acunzo RS. Pseudo 2:1 atrioventricular block and T wave alternans in the long QT syndromes. J Am Coll Cardiol 1991;18(05):1363-1366
3 Patel C, Narayanaswamy S, Medina-Ravell VA, Yan GX. A rare cause of 2:1 AV block: long QT syndrome. J Cardiovasc Electrophysiol 2008;19(09):990

4 Martí-Almor J, Berrueco R, García-Algar O, et al. QT interval in newborns of different ethnic origin: usefulness of neonatal ECG screening. Rev Esp Cardiol 2008;61(09):980-982 